\title{
Bull's eye grating integrated with optical nanoantennas for plasmonic enhancement of graphene long-wave infrared photodetectors
}

Cite as: Appl. Phys. Lett. 114, 091108 (2019); https://doi.org/10.1063/1.5082664

Submitted: 22 November 2018 . Accepted: 24 February 2019 . Published Online: 08 March 2019

Nima Sefidmooye Azar (D), Vivek Raj Shrestha (D), and Kenneth B. Crozier (D)
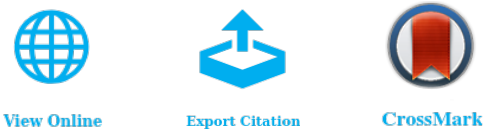

\section{ARTICLES YOU MAY BE INTERESTED IN}

High-responsivity and polarization-discriminating terahertz photodetector based on plasmonic resonance

Applied Physics Letters 114, 091105 (2019); https://doi.org/10.1063/1.5085813

A critical review of recent progress on negative capacitance field-effect transistors

Applied Physics Letters 114, 090401 (2019); https://doi.org/10.1063/1.5092684

Realization and characterization of terahertz surface plasmon light capsules

Applied Physics Letters 114, 091110 (2019); https://doi.org/10.1063/1.5085862
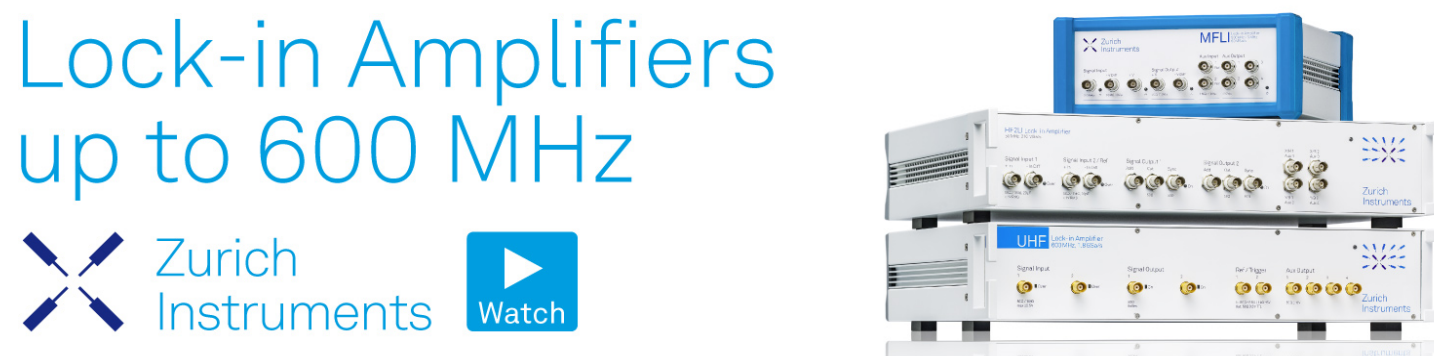


\title{
Bull's eye grating integrated with optical nanoantennas for plasmonic enhancement of graphene long-wave infrared photodetectors
}

\author{
Cite as: Appl. Phys. Lett. 114, 091108 (2019); doi: 10.1063/1.5082664 \\ Submitted: 22 November 2018 - Accepted: 24 February 2019 . \\ Published Online: 8 March 2019
}

Nima Sefidmooye Azar, ${ }^{1}$ (D) Vivek Raj Shrestha, ${ }^{2}$ (D) and Kenneth B. Crozier ${ }^{1,2, a)}$ (iD

\begin{abstract}
AFFILIATIONS
${ }^{7}$ Department of Electrical and Electronic Engineering, University of Melbourne, Melbourne, Victoria 3010, Australia

${ }^{2}$ School of Physics, University of Melbourne, Melbourne, Victoria 3010, Australia
\end{abstract}

a) Author to whom correspondence should be addressed: kcrozier@unimelb.edu.au

\begin{abstract}
Two-dimensional (2D) materials have exhibited potential for infrared detection at room temperature, yet their low light absorption impedes their widespread application. In addition, micromechanical cleavage, which is the main method by which high-quality $2 \mathrm{D}$ layers are achieved, typically leads to small-area flakes, hampering their application as photodetectors. In this work, we designed a hybrid plasmonic structure, comprising a metallic bull's eye grating and optical nanoantennas, to collect and concentrate light into a piece of single-layer graphene with sub-wavelength lateral extent. This boosts the interaction between the graphene and light, thereby improving its photodetection performance in the technologically important long-wave infrared (LWIR) region. Finite-difference time-domain electromagnetic simulations were performed to this end. The plasmonic structure we present is predicted to enhance the absorption of light by the graphene by $\sim 558$ times, which in turn is predicted to enhance the detectivity of the LWIR photodetector by $\sim 32$ times.
\end{abstract}

Published under license by AIP Publishing. https://doi.org/10.1063/1.5082664

Infrared (IR) photodetectors with high performance at room temperature are in high demand, having widespread applications that include night vision, security, search and rescue, thermography, and astronomy. ${ }^{1}$ Long-wave IR (LWIR, $8-12 \mu \mathrm{m}$ ) is particularly interesting for thermal imaging since the peak emission of objects at room temperature lies in this range. ${ }^{2}$ Currently, LWIR photodetection technology is dominated by mercury-cadmium-telluride (MCT). ${ }^{3}$ Severe thermal generation of charge carriers at the small LWIR energies leads to poor, noisy performance of MCT semiconductors at room temperature, necessitating the use of costly and bulky cryogenic systems. In addition, MCT detectors are fabricated by a complex and high-cost process. ${ }^{4}$

Owing to their appealing electrical, optical, mechanical, and chemical properties, two-dimensional (2D) materials have recently attracted a great deal of interest as the potential building blocks of the next generation electronic and optoelectronic devices. ${ }^{5,6}$ Furthermore, it has been suggested that their thin nature could allow infrared detectors with lower noise levels than those of conventional bulk semiconductors. ${ }^{7}$ Accordingly, 2D materials such as graphene, ${ }^{8,9}$ black phosphorous, ${ }^{10,11}$ black phosphorus-arsenic, ${ }^{7}$ tellurium, ${ }^{12}$ and platinum diselenide ${ }^{13}$ have been explored for infrared detection.

One major obstacle for photodetectors based on 2D materials, however, is that such materials have inherently low absorption due to their atomic thinness. This can result in low photoresponsivity. ${ }^{14}$ Monolayer graphene, for instance, absorbs only 2.3\% of the incident visible light. ${ }^{15}$ To overcome this issue and enhance the light-matter interaction in 2D materials, various optical techniques such as integration with plasmonic structures, ${ }^{14,16-19}$ optical cavities, ${ }^{12,20,21}$ and optical waveguides ${ }^{22,23}$ have been demonstrated. Yao et al., for example, used an array of nanobar antennas with nanoscale gaps to enhance the performance of mid-wave infrared graphene photodetectors. ${ }^{14}$ Another problem is that the high-quality 2D flakes produced by the mechanical exfoliation of layered materials are generally of small lateral dimensions, which results from the fragmentation effects inherent to this procedure ${ }^{24}$ and is unfavorable for photodetection applications.

Herein, we addressed the aforementioned issues by employing a composite plasmonic structure consisting of a metallic bull's eye grating at whose center there is an aperture containing an array of optical nanoantennas. The schematic diagrams of the proposed LWIR photodetector are provided in Figs. 1(a) and 1(b). The diffraction grating collects the incident light from a large area, couples it into surface plasmon polaritons (SPPs), ${ }^{25}$ and concentrates them onto the small-area $2 \mathrm{D}$ material in the device center (i.e., at the aperture). The grating can thus be thought of as a plasmonic lens that improves the detectivity, as discussed later. Bull's eye gratings have been used in high-speed 


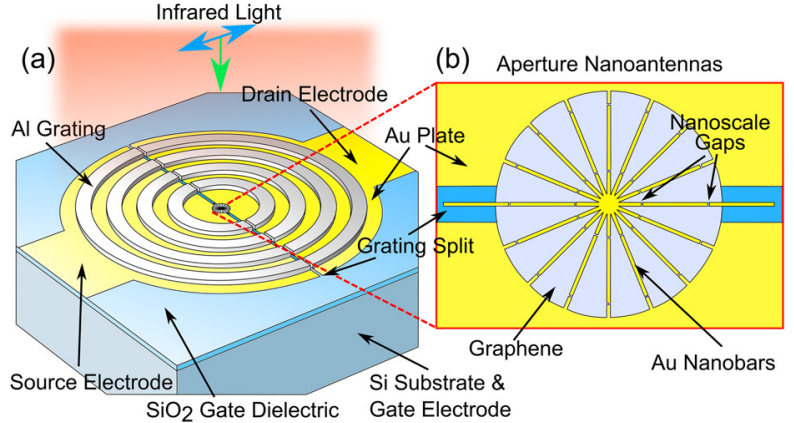

subwavelength photodetectors based on conventional semiconductors. ${ }^{26-28}$ The aperture nanoantenna array is a polarization-independent arrangement of nanobar antennas separated by nanoscale gaps. These enhance the electromagnetic field in the near-field zone, particularly in the gaps, through localized surface plasmon resonances (LSPRs). ${ }^{29}$ This enhances the light matter interaction in the underlying $2 \mathrm{D}$ material. We expect that this approach can be applied to many different 2D materials. Here, we considered the specific case of monolayer graphene.

In this study, the emphasis is not on the experimental realization. Nonetheless, the fabrication sequence could begin with the exfoliation of monolayer graphene onto an $\mathrm{SiO}_{2} / \mathrm{Si}$ substrate. This would be followed by the fabrication of the gold plate, aperture nanoantennas, and aluminum ridges via standard lithography/metal evaporation/lift-off processes. In this design, the bull's eye structure is split into two halves, across which a source/drain bias can be applied to collect the photocurrent. The Si substrate acts as the gate electrode and the $\mathrm{SiO}_{2}$ film as the gate dielectric of this phototransistor. Applying a gate voltage could allow the photodetection process to be enhanced by manipulating the Fermi level of the graphene. The geometrical parameters of the grating and aperture nanoantennas, as illustrated in Figs. 1 (c) and 1(d), respectively, were optimized for a wavelength of $\lambda=10.84 \mu \mathrm{m}$. The structural parameters are as follows: $p_{1}=10.5 \mu \mathrm{m}, p_{2}=0.5 p_{1}=5.25 \mu \mathrm{m}, h_{2}=0.16 p_{1}=1.68 \mu \mathrm{m}$, $d=1.25 p_{1}=13.125 \mu \mathrm{m}, \quad r=3.14 \mu \mathrm{m}, R=55.125 \mu \mathrm{m}, \quad h_{1}=30 \mathrm{~nm}$, $N=4, s=500 \mathrm{~nm}, l=1.8 \mu \mathrm{m}, w=100 \mathrm{~nm}, g=40 \mathrm{~nm}$, thickness of the gold nanoantennas $=30 \mathrm{~nm}$, and $\mathrm{SiO}_{2}$ thickness $=300 \mathrm{~nm}$.

Maxwell's equations were solved utilizing the finite-difference time-domain $^{30}$ (FDTD) technique implemented in the software package FDTD Solutions. ${ }^{31}$ The light source was a linearly polarized totalfield scattered-field (TFSF) plane wave at normal incidence. The mesh size was $5 \mathrm{~nm}$ around the finest features. The simulation environment was a cuboid with boundaries at least half the largest simulated wavelength away from any scatterer. Perfectly matched layers (PMLs) were used to describe the boundary conditions.

The optical absorption of graphene arises from both intraband and interband transitions. ${ }^{32,33}$ We modeled graphene as a conductive sheet with a surface conductivity $\sigma=\sigma_{\text {intra }}+\sigma_{\text {inter }}$ which can be expressed as ${ }^{34}$

$$
\begin{aligned}
\sigma\left(\omega, \epsilon_{f}, \Gamma, T\right)= & \frac{j q^{2}(\omega-j 2 \Gamma)}{\pi \hbar^{2}}\left[\frac{1}{(\omega-j 2 \Gamma)^{2}} \int_{0}^{\infty} \epsilon\left(\frac{\partial f(\epsilon)}{\partial \epsilon}-\frac{\partial f(-\epsilon)}{\partial \epsilon}\right)\right. \\
& \left.\times d \epsilon-\int_{0}^{\infty} \frac{f(-\epsilon)-f(\epsilon)}{(\omega-j 2 \Gamma)^{2}-4(\epsilon / \hbar)^{2}} d \epsilon\right]
\end{aligned}
$$

where $\omega$ denotes the angular frequency, $\epsilon_{f}$ the Fermi level, $\Gamma$ the scattering rate, $T$ the temperature, $q$ the elementary charge, $\hbar$ the reduced Planck constant, $\epsilon$ the energy, and $f(\epsilon)=\left(\exp \left[\left(\epsilon-\epsilon_{f}\right) / k_{B} T\right]+1\right)^{-1}$ the Fermi-Dirac distribution in which $k_{B}$ is the Boltzmann constant. The values for $\epsilon_{f}$ and $\Gamma$ were set to $0.341 \mathrm{eV}$ and $0.0066 \mathrm{eV}$, respectively, which were taken from Ref. 35 . The optical power absorbed in graphene was obtained by calculating the Joule losses.

Figure 2 plots the power (normalized by the source power) absorbed in a graphene ( $\mathrm{Gr}$ ) layer sitting on an $\mathrm{SiO}_{2}$ substrate (as in Ref. 35) and in a suspended graphene. In agreement with the experimental results from Ref. 35, this figure indicates that the absorption in graphene declines in the short-wave IR region due to the Pauli blocking of interband transitions and begins to rise in LWIR as the intraband transitions come into play. This figure also suggests that the absorption in the graphene on the $\mathrm{SiO}_{2}$ substrate is lower than the suspended one. This absorption reduction in $2 \mathrm{D}$ materials in the presence of a substrate comes from the destructive interference between the incident and reflected waves. ${ }^{36}$

To evaluate the proposed device and determine the contribution from each plasmonic component, four different cases were considered: (a) "No Plasmonics," where the graphene sits on the $\mathrm{SiO}_{2} / \mathrm{Si}$ substrate without any plasmonic structures; (b) "Grating," in which the gold plate and the aluminum ridges are added to the "No Plasmonics" configuration; (c) "Nanoantennas," in which the gold plate (without aluminum ridges) and the aperture nanoantennas are added to the "No Plasmonics" configuration; and (d) "Nanoantennas and Grating,"

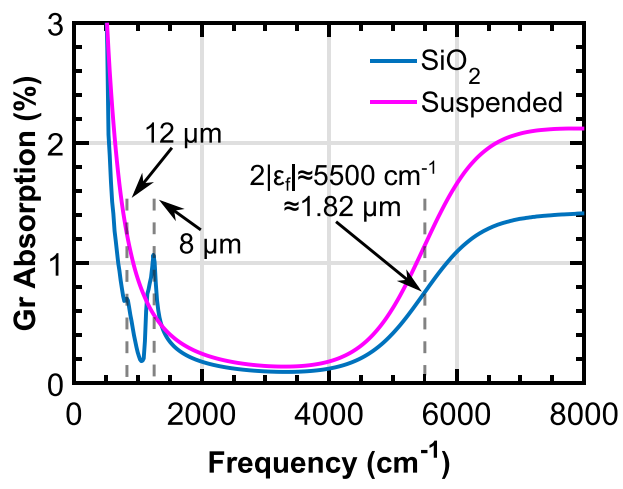

FIG. 2. Normalized power absorbed in the graphene sitting on an $\mathrm{SiO}_{2}$ substrate and in the suspended graphene. In both cases, graphene has $\epsilon_{f}=0.341 \mathrm{eV}$ and $\Gamma=0.0066 \mathrm{eV}$. 

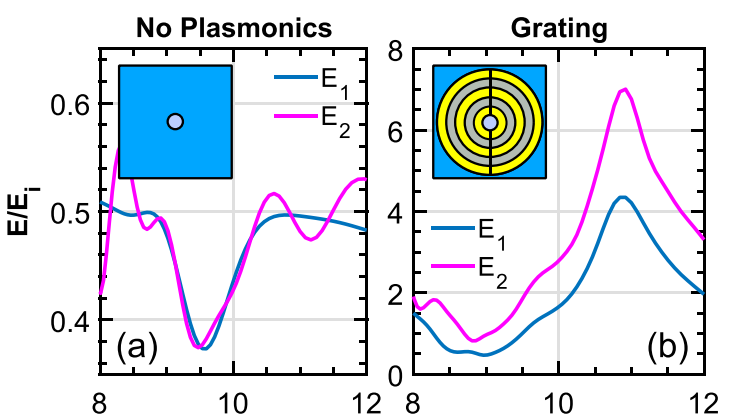

Wavelength $(\mu \mathrm{m})$

Nanoantennas

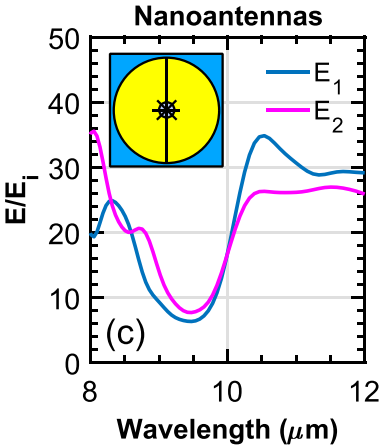

Wavelength $(\mu \mathrm{m})$
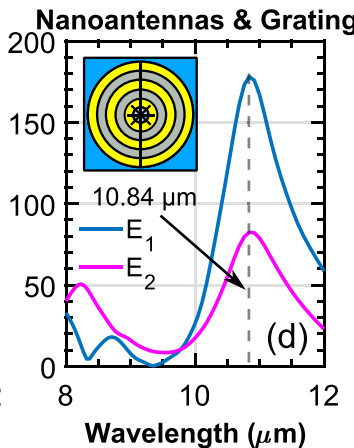

FIG. 3. (a)-(d) Electric field enhancement at points $E_{1}$ and $E_{2}$ in the "No Plasmonics," "Grating," "Nanoantennas," and "Nanoantennas and Grating" configurations, respectively. $E_{i}$ is the norm of the incident electric field.

where all the components (gold plate, aluminum ridges, and aperture nanoantennas) are present.

Figure 3 presents the electric field enhancement at points $E_{1}$ and $E_{2}$, whose locations are defined in Fig. 1(d), for the four cases mentioned above. It also depicts cartoons of the configurations in the inset. The electric field is monitored at the middle of the nanoscale gaps on the graphene surface, and its norm is reported here. $\mathrm{E}_{i}$ represents the norm of the incident electric field. We observe an electric field enhancement of $\sim 180$ and $\sim 80$ times at the $\mathrm{E}_{1}$ and $\mathrm{E}_{2}$ gaps, respectively, in the "Nanoantennas and Grating" configuration at the resonance wavelength.

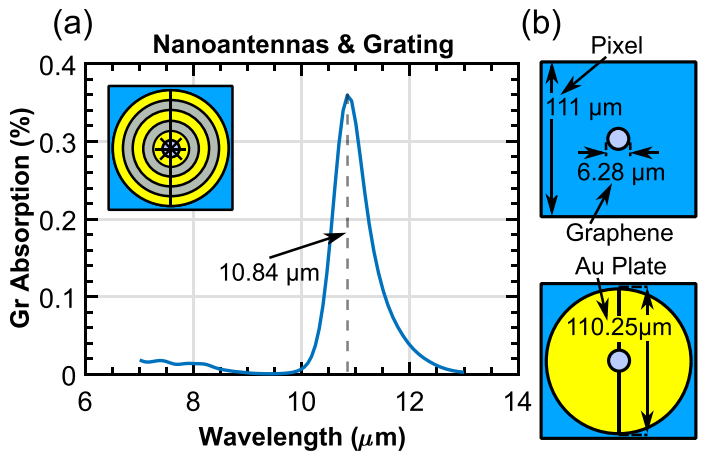

FIG. 4. (a) Normalized power absorbed in graphene as a function of the wavelength in the "Nanoantennas and Grating" structure. (b) Sketch showing the pixel, gold plate, and graphene size.

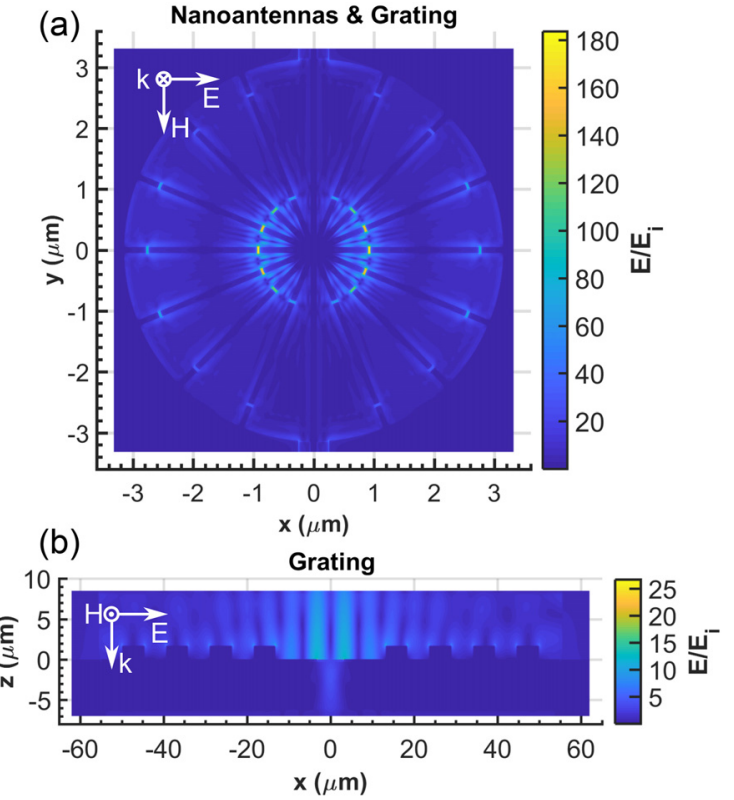

FIG. 5. Electric field enhancement distribution at $\lambda=10.84 \mu \mathrm{m}$ in (a) the aperture of the "Nanoantennas and Grating" and (b) the cross section of the "Grating" structure. $E_{i}$ is the norm of the incident electric field.

Figure 4(a) graphs the normalized power absorbed in graphene as a function of the wavelength in the "Nanoantennas and Grating" structure. The power absorbed in graphene is normalized to the power incident through a square window with a side length of $111 \mu \mathrm{m}$, which we refer to as the "pixel." As illustrated in Fig. 4(b), the graphene and the gold plate have diameters of 6.28 and $110.25 \mu \mathrm{m}$, respectively. Here, the graphene absorption is $0.36 \%$ at the resonance wavelength $(\lambda=10.84 \mu \mathrm{m})$. For the same configuration, Fig. 5(a) depicts the electric field enhancement profile in the bull's eye aperture at $\lambda=10.84 \mu \mathrm{m}$. These results suggest that the proposed architecture provides two sets of locations at which field enhancement occurs, i.e., $E_{1}$ and $E_{2}$. A cross-sectional view of the electric field enhancement profile in the "Grating" structure is displayed in Fig. 5(b) at $\lambda=10.84 \mu \mathrm{m}$, which shows the considerable field enhancement in the aperture region provided by the metallic grating.

TABLE I. Absorption enhancement provided by the plasmonic structures at $\lambda=10.84 \mu \mathrm{m}$.

\begin{tabular}{lcc}
\hline \hline & $\begin{array}{c}\text { Gr absorption } \\
(\%)^{\mathrm{a}}\end{array}$ & $\begin{array}{c}\text { Absorption } \\
\text { enhancement }\end{array}$ \\
\hline No plasmonics & 0.0006 & $\ldots$ \\
Grating & 0.0291 & 45.17 \\
Nanoantennas & 0.0168 & 26.10 \\
Nanoantennas and Grating & 0.3595 & 558.33
\end{tabular}

${ }^{\mathrm{a}}$ Gr Absorption $=$ Power Absorbed in Graphene [circle, diameter $\left.=6.28 \mu \mathrm{m}\right]$

Source Power $[$ square, side length $=111 \mu \mathrm{m}]$

${ }^{\mathrm{b}}$ Absorption Enhancement $=\frac{\text { Gr Absorption [With Plasmonics] }}{\text { Gr Absorption [No Plasmonics] }}$. 
TABLE II. Collection efficiency and detectivity enhancement provided by the plasmonic structures at $\lambda=10.84 \mu \mathrm{m}$.

\begin{tabular}{|c|c|c|}
\hline & Gr absorption (\%) & Collection efficiency (\%) \\
\hline No plasmonics-LAG & 0.1985 & $\ldots$ \\
\hline Grating & 0.0291 & 14.65 \\
\hline Nanoantennas & 0.0168 & 8.47 \\
\hline \multirow[t]{2}{*}{ Nanoantennas and Grating } & 0.3595 & 181.16 \\
\hline & Detectivity enhancement ${ }^{\mathrm{b}}$ & \\
\hline No plasmonics-LAG & $\ldots$ & \\
\hline Grating & 2.57 & \\
\hline Nanoantennas & 1.49 & \\
\hline Nanoantennas and Grating & 31.80 & \\
\hline
\end{tabular}

Table I lists the absorption enhancement provided by the various cases of the plasmonic structure. Here, the absorption enhancement is defined as the graphene absorption ratio between the configurations including plasmonics and the "No Plasmonics" one. We find that the "Nanoantennas and Grating" architecture leads to an absorption enhancement of $\sim 558$ times.

An important figure of merit of a photodetector is its detectivity $\left(D^{*}\right)$ which represents its ability to detect the signal from noise. In the case of optical immersion where the light-absorbing material is coupled to the incident radiation by an optical concentrator, the detectivity is given by ${ }^{37}$

$$
D^{*}=\frac{\sqrt{A_{O} \Delta f}}{N E P}=\frac{q \lambda \eta \gamma}{h c} \frac{\sqrt{A_{O} \Delta f}}{\sqrt{\overline{I_{n}^{2}}}},
$$

where $A_{O}$ is the optical area of the concentrator, $\Delta f$ the detector bandwidth, NEP the noise equivalent power, $q$ the elementary charge, $\lambda$ the wavelength, $\eta$ the external quantum efficiency, $\gamma$ the photoelectric gain, $h$ the Planck constant, $c$ the speed of light, and $I_{n}$ the noise current. Assuming that the noise is dominated by the thermal generation and recombination of carriers, the noise current can be expressed as ${ }^{37}$ $\overline{I_{n}^{2}}=2 q^{2} \gamma^{2}(g+r) \Delta f$, in which $g$ and $r$ denote the thermal generation and recombination rates, respectively. Consequently, one can express the detectivity as

$$
D^{*}=\frac{\lambda}{h c \sqrt{2(G+R)}} \sqrt{\frac{A_{O}}{A_{D}}} \frac{\eta}{\sqrt{t}},
$$

where $G$ and $R$ stand for the volume rates of the thermal generation and recombination, respectively, and $A_{D}$ and $t$ denote the area and thickness of the photoactive material. This expression implies that the detectivity can be boosted by increasing the $A_{O} / A_{D}$ ratio (i.e., optical immersion ${ }^{37}$ ), increasing $\eta$ (by enhancing the light absorption and the photocarrier generation and collection efficiency), and reducing the material thickness (by using 2D materials rather than bulk semiconductors).

To investigate the collection efficiency and detectivity enhancement of the proposed plasmonic structure, we compared it with a device without plasmonics for which the graphene has the same area as the optical concentrator (circle, diameter $=110.25 \mu \mathrm{m}$ ). This is referred to as "No Plasmonics-LAG (large-area graphene)." Here, we define the collection efficiency as the ratio of the absorption in the graphene in the device under question to that in the "No PlasmonicsLAG" configuration, as this ratio represents the ability of the plasmonic structures to concentrate light. The detectivity $\left(D^{*}\right)$ enhancement of a particular device is defined as its detectivity divided by that of the "No Plasmonics-LAG" configuration. From Eq. (3), it follows that this is given by

$$
D^{*} \text { Enhancement }=\sqrt{\frac{A_{O}}{A_{D}}} \times \text { Collection Efficiency, }
$$

as $A_{O} / A_{D}=1$ for "No Plasmonics-LAG" and $\eta$ is proportional to graphene absorption. The results are reported in Table II. The "Nanoantennas and Grating" configuration gives rise to a detectivity enhancement of $\sim 32$ times. It should also be noted that the latter equation for detectivity enhancement holds true for any noise source whose current is proportional to $\sqrt{A_{D} \Delta f}$.

To conclude, here we demonstrated how two plasmonic phenomena, namely, surface plasmon polaritons and localized surface plasmons, can be integrated and exploited to enhance the light-matter interaction and photodetection performance in $2 \mathrm{D}$ materials. The presented plasmonic architecture leads to significant light absorption and detectivity enhancement for LWIR photodetectors. Graphene portrayed the 2D material here, yet the introduced method should work as well for other thin $2 \mathrm{D}$ materials.

This work was supported by the Defense Advanced Research Projects Agency under Contract No. HR0011-16-1-0004 and the Australian Research Council (DP180104141 and FT140100577).

\section{REFERENCES}

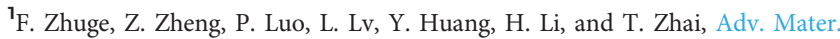
Technol. 2, 1700005 (2017).

${ }^{2}$ M. Razeghi and B. M. Nguyen, Rep. Prog. Phys. 77, 082401 (2014).

${ }^{3}$ P. Norton, Opto-Electron. Rev. 10, 159-174 (2002).

${ }^{4}$ A. V. Barve, S. J. Lee, S. K. Noh, and S. Krishna, Laser Photonics Rev. 4, 738-750 (2010). 
${ }^{5}$ C. Tan, X. Cao, X. J. Wu, Q. He, J. Yang, X. Zhang, J. Chen, W. Zhao, S. Han, G. H. Nam, M. Sindoro, and H. Zhang, Chem. Rev. 117, 6225-6331 (2017).

${ }^{6}$ F. H. L. Koppens, T. Mueller, P. Avouris, A. C. Ferrari, M. S. Vitiello, and M. Polini, Nat. Nanotechnol. 9, 780-793 (2014).

${ }^{7}$ M. Amani, E. Regan, J. Bullock, G. H. Ahn, and A. Javey, ACS Nano 11, 11724-11731 (2017).

${ }^{8}$ M. Freitag, T. Low, W. Zhu, H. Yan, F. Xia, and P. Avouris, Nat. Commun. 4, 1951 (2013).

${ }^{9}$ N. Liu, H. Tian, G. Schwartz, J. B. H. Tok, T. L. Ren, and Z. Bao, Nano Lett. 14, 3702-3708 (2014).

${ }^{10}$ X. Chen, X. Lu, B. Deng, O. Sinai, Y. Shao, C. Li, S. Yuan, V. Tran, K. Watanabe, T. Taniguchi, D. Naveh, L. Yang, and F. Xia, Nat. Commun. 8, 1672 (2017).

"Q. Guo, A. Pospischil, M. Bhuiyan, H. Jiang, H. Tian, D. Farmer, B. Deng, C. Li, S. J. Han, H. Wang, Q. Xia, T. P. Ma, T. Mueller, and F. Xia, Nano Lett. 16, 4648-4655 (2016).

${ }^{12}$ M. Amani, C. Tan, G. Zhang, C. Zhao, J. Bullock, X. Song, H. Kim, V. R. Shrestha, Y. Gao, K. B. Crozier, M. Scott, and A. Javey, ACS Nano 12, 7253-7263 (2018)

${ }^{13}$ X. Yu, P. Yu, D. Wu, B. Singh, Q. Zeng, H. Lin, W. Zhou, J. Lin, K. Suenaga, Z. Liu, and Q. J. Wang, Nat. Commun. 9, 1545 (2018).

${ }^{14}$ Y. Yao, R. Shankar, P. Rauter, Y. Song, J. Kong, M. Loncar, and F. Capasso, Nano Lett. 14, 3749-3754 (2014).

${ }^{15}$ R. R. Nair, P. Blake, A. N. Grigorenko, K. S. Novoselov, T. J. Booth, T. Stauber, N. M. R. Peres, and A. K. Geim, Science 320, 1308 (2008).

${ }^{16}$ C. Chakraborty, R. Beams, K. M. Goodfellow, G. W. Wicks, L. Novotny, and A. N. Vamivakas, Appl. Phys. Lett. 105, 241114 (2014).

${ }^{17}$ Z. Chen, X. Li, J. Wang, L. Tao, M. Long, S. J. Liang, L. K. Ang, C. Shu, H. K. Tsang, and J. B. Xu, ACS Nano 11, 430-437 (2017).

${ }^{18}$ Z. Fang, Z. Liu, Y. Wang, P. M. Ajayan, P. Nordlander, and N. J. Halas, Nano Lett. 12, 3808-3813 (2012)

${ }^{19}$ J. Lin, H. Li, H. Zhang, and W. Chen, Appl. Phys. Lett. 102, 203109 (2013).

${ }^{20}$ M. Engel, M. Steiner, A. Lombardo, A. C. Ferrari, H. V. Löhneysen, P. Avouris, and R. Krupke, Nat. Commun. 3, 906 (2012).
${ }^{21}$ M. Furchi, A. Urich, A. Pospischil, G. Lilley, K. Unterrainer, H. Detz, P. Klang, A. M. Andrews, W. Schrenk, G. Strasser, and T. Mueller, Nano Lett. 12, 2773-2777 (2012).

${ }^{22}$ N. Youngblood, C. Chen, S. J. Koester, and M. Li, Nat. Photonics 9, 247-252 (2015).

${ }^{23}$ C. Chen, N. Youngblood, R. Peng, D. Yoo, D. A. Mohr, T. W. Johnson, S.-H. Oh, and M. Li, Nano Lett. 17, 985-991 (2017).

${ }^{24} \mathrm{M}$. Yi and Z. Shen, J. Mater. Chem. A 3, 11700-11715 (2015).

${ }^{25} \mathrm{E}$. Wijaya, C. Lenaerts, S. Maricot, J. Hastanin, S. Habraken, J. P. Vilcot, R. Boukherroub, and S. Szunerits, Curr. Opin. Solid State Mater. Sci. 15, 208-224 (2011).

${ }^{26}$ F. F. Ren, K. W. Ang, J. Ye, M. Yu, G. Q. Lo, and D. L. Kwong, Nano Lett. 11, 1289-1293 (2011).

${ }^{27}$ T. Ishi, J. Fujikata, K. Makita, T. Baba, and K. Ohashi, Jpn. J. Appl. Phys. Part 2 44, L 364-L 366 (2005).

${ }^{28}$ R. D. R. Bhat, N. C. Panoiu, S. R. J. Brueck, and R. M. Osgood, Opt. Express 16, 4588-4596 (2008).

${ }^{29}$ V. Giannini, A. I. Fernández-Domínguez, S. C. Heck, and S. A. Maier, Chem. Rev. 111, 3888-3912 (2011).

${ }^{30} \mathrm{~A}$. Taflove and S. C. Hagness, Computational Electrodynamics: The FiniteDifference Time-Domain Method (Artech House, 2005).

${ }^{31}$ See http://www.lumerical.com/tcad-products/fdtd/ for "Lumerical Inc."

${ }^{32}$ H. Choi, F. Borondics, D. A. Siegel, S. Y. Zhou, M. C. Martin, A. Lanzara, and R. A. Kaindl, Appl. Phys. Lett. 94, 172102 (2009).

${ }^{33}$ J. M. Dawlaty, S. Shivaraman, J. Strait, P. George, M. Chandrashekhar, F. Rana, M. G. Spencer, D. Veksler, and Y. Chen, Appl. Phys. Lett. 93, 131905 (2008).

${ }^{34}$ G. W. Hanson, J. Appl. Phys. 103, 064302 (2008).

${ }^{35}$ H. Yan, F. Xia, W. Zhu, M. Freitag, C. Dimitrakopoulos, A. A. Bol, G. Tulevski, and P. Avouris, ACS Nano 5, 9854-9860 (2011).

${ }^{36}$ H. Fang, H. A. Bechtel, E. Plis, M. C. Martin, S. Krishna, E. Yablonovitch, and A. Javey, Proc. Natl. Acad. Sci. U. S. A. 110, 11688-11691 (2013).

${ }^{37}$ J. Piotrowski, Opto-Electron. Rev. 12, 111-122 (2004). 